\title{
A system for preoperative stratification of cataract patients according to risk of intraoperative complications: a prospective analysis of 1441 cases
}

\author{
M Muhtaseb, A Kalhoro, A lonides
}

Br J Ophthalmol 2004;88:1242-1246. doi: 10.1136/bjo.2004.046003

\begin{abstract}
Aim: To devise a simple, robust scoring system for assessing the risk of intraoperative complications in patients undergoing cataract surgery.

Methods: 1441 consecutive patients undergoing phacoemulsification cataract surgery were assessed preoperatively according to weighted criteria. According to the points of risk they accumulated using this system, the patients were preoperatively allocated to one of four risk groups. Data were prospectively collected on the occurrence of intraoperative complications and entered into a computerised database. The total rate of intraoperative complications for each risk group as well as the rate of each reported complication for each risk group were calculated.

Results: The rate of intraoperative complications increased in frequency through the risk groups: $1=4.32 \%, 2=7.45 \%$, $3=13.48 \%$, and $4=32.00 \%(p<0.001)$. Furthermore, the following complications also increased in frequency through the risk groups in their own right $(p<0.05$ in each case): posterior capsule rupture, vitreous loss, incomplete capsulorrhexis, zonule dehiscence, wound burn/leak, and lost nuclear fragment into vitreous cavity.

Conclusion: These results suggest that candidates for cataract surgery can be simply and uniformly assessed preoperatively and categorised to a "risk group" according to their risk of intraoperative complications. This allows for: (1) individualised counselling on the chances of operative complications, (2) meaningful comparisons between national complication rates and those of individual units or surgeons, and (3) better selection of cases suitable for instruction.
\end{abstract}

$\mathrm{T}$ he providers of health care and those who receive it share an interest in the results of surgical procedures. According to the UK Department of Health ${ }^{1}$ over 247000 cataract operations were performed in the year 2001-2. This was surpassed only by upper gastrointestinal endoscopy, cystoscopy, and childbirth. On census day 2001 the population of the United Kingdom was 58.8 million, ${ }^{2}$ so it is possible to calculate that the rate of cataract surgery at that time was 41.32/10 000 population.

In the United States, where the estimated current population is 291.3 million people, ${ }^{3}$ the National Survey of Ambulatory Surgery (NSAS) collected data on all outpatient procedures carried out until 1996. In 1996, extraction of the lens was the most frequently performed outpatient procedure with 2.367 million operations carried out. ${ }^{4}$ The NSAS gave the rate of cataract surgery in 1996 as 89.6/10 000 population. This was by far the most frequently performed outpatient procedure. It also surpassed all inpatient procedures in frequency.
National data have been collected on cataract surgery in the United Kingdom on two occasions ${ }^{56}$ and the rate of intraoperative complications was found to be $7 \%{ }^{5}$ and $7.5 \%$, respectively. In the first survey, the authors did not specify what intraoperative events were counted as a "complication" although they did mention that $4 \%$ of all patients had capsule rupture without vitreous loss and a further $1 \%$ had capsule rupture associated with vitreous loss. ${ }^{5}$ The second survey recorded the following intraoperative events: anterior chamber haemorrhage, anterior chamber collapse, torn iris, iris emulsification, persistent iris prolapse, choroidal haemorrhage, capsule rupture and vitreous loss, incomplete cortical clean up, loss of nuclear fragment into vitreous, loss of intraocular lens into vitreous, torn Descemet's membrane, and abnormality in wound closure. The rate of capsule rupture and vitreous loss was $4.4 \%$, with all other events occurring at a frequency of between $0.07 \%$ and $1 \%{ }^{6}$

Patients being prepared for cataract surgery in our unit are informed that the procedure carries a risk of about $5 \%$ that an unplanned event may occur. Similar information is provided to patients wherever informed consent is obtained. However, not all cataract operations present the same degree of complexity. It follows that the rate of complications is likely to be higher in more complex cataract cases than in more straightforward ones. We thought it would be valuable to be able to uniformly and objectively assess the risk of complications in individual patients preoperatively (for example, at the time of scheduling surgery).

Such a system of patient classification is the subject of this report.

\section{MATERIALS AND METHODS}

The approval of the ethics review board of our hospital was sought and obtained.

We conducted a Medline review of the literature pertaining to complications during phacoemulsification cataract surgery in order to identify those characteristics that have been shown to increase the risk of intraoperative complications. ${ }^{7-16}$ The most consistently reported risk factors were chosen to form the basis of a newly devised weighted scoring system (table 1). There was no scheme for weighting the reviewed papers to determine their influence on the system.

A brief summary of our findings follows.

Kuchle et al $^{7}$ showed that anterior chamber (AC) depth of less than $2.5 \mathrm{~mm}$ was associated with an intraoperative complication rate of $13.4 \%$ compared with a rate of $2.8 \%$ when AC depth was $2.5 \mathrm{~mm}$ or greater in 174 eyes with pseudoexfoliation (a 4.79-fold increase). These figures relate to zonular dialysis and/or vitreous loss. Psuedoexfoliation itself was shown to be associated with a 2.6-fold increase in

Abbreviations: $E C C E$, extracapsular cataract extraction; $I O L$, intraocular lens; PCR, posterior capsule rupture 


\begin{tabular}{|c|c|c|}
\hline Category A (no points) & Category B (1 point each) & Category C ( 3 points each) \\
\hline $\begin{array}{l}\text { No additional risk factors } \\
\text { carried by the patient }\end{array}$ & $\begin{array}{l}\text { Previous vitrectomy } \\
\text { Corneal scarring } \\
\text { Small pupil }(<3 \mathrm{~mm}) \\
\text { Shallow anterior chamber } \\
\text { (depth }<2.5 \mathrm{~mm} \text { ) } \\
\text { Age }>88 \text { years } \\
\text { High ametropia ( }>6 \text { D of myopia or } \\
\text { hyperopia) } \\
\text { Posterior capsule plaque } \\
\text { Posterior polar cataract } \\
\text { Miscellaneous risks assessed by the } \\
\text { surgeon (eg, poor position of eye/patient) }\end{array}$ & $\begin{array}{l}\text { Dense/total/white or brunescent } \\
\text { cataract } \\
\text { Pseudoexfoliation } \\
\text { Phacodonesis }\end{array}$ \\
\hline
\end{tabular}

the rate of capsular/zonular tear or vitreous loss by Drolsum et al. ${ }^{8}$ In their study, $9.6 \%$ of 164 eyes with pseudoexfoliation experienced a complication compared with $3.7 \%$ of 916 eyes without pseudoexfoliation. The vast majority of these cataract extractions were carried out by phacoemulsification $(96.2 \%)$. Phacoemulsification in eyes with white cataract has been shown to be associated with incomplete capsulorrhexis, posterior capsule rupture, and conversion to manual nonphacoemulsification technique. ${ }^{10}$ Singh et al demonstrated an elevated rate of wound site thermal injury in brunescent cataracts undergoing phacoemulsification. ${ }^{11}$

In their prospective study of 23 vitrectomised eyes undergoing phacoemulsification, Lacalle et $a^{12}$ noted a higher than usual incidence of surgical problems and complications including poor pupil dilatation (seven eyes with pupils $<3 \mathrm{~mm}$ ), sudden changes in the anterior chamber depth and pupil size ( 6 eyes), and unusual flaccidity and mobility of the posterior capsule (six eyes) which was associated with posterior capsule plaque and age $<50$ years. Posterior capsule tears occurred in two eyes $(8.7 \%)$. Pinter and Sugar ${ }^{13}$ demonstrated that vitrectomised eyes presented a higher incidence of posterior subcapsular cataract than controls, and that these posed challenges during cataract surgery, including unstable posterior capsules, loose zonules, and posterior capsule plaques that could not be removed.

Phacoemulsification in eyes with posterior polar cataract is associated with posterior capsule rupture in $26 \%-36 \%$ of cases. $^{14}{ }^{15}$ Intraoperative complications such as posterior capsule tears, vitreous loss, and loss of the nucleus occurred in $10 \%$ of patients aged over 88 years compared to only $3 \%$ in those aged less than 88 years in a study by Berler. ${ }^{16}$ In addition, the elderly were more likely to have needed pupil stretching and were much less likely to achieve a visual acuity of $6 / 12$ or better if a complication had occurred compared to the younger group (40\% versus $90.5 \%$ ).

Potential risk factors for intraoperative complications that were not consistently shown to be associated with a higher rate of complications (and so were omitted from the scoring system) included glaucoma, diabetes mellitus, and previous scleral buckling.

Following the literature review we formulated three categories of characteristics relating to (1) the patient or (2) the eye scheduled for operation (see table 1 above). We allocated points to each risk factor according to its potential for increasing surgical risk.

A data sheet was attached to the case notes and, at the preoperative ward round, the surgeon would indicate the presence of any risk factors. Each patient was categorised into a risk group according to the number of points they accrued: group 1 (no added risk) 0 points; group 2 (low risk) 1-2 points; group 3 (moderate risk) 3-5 points; group 4 (high risk) 6 points or more.
The thresholds for transition between risk groups were decided upon empirically.

Once the operation had been completed, the following data were recorded on the data sheet: date of surgery, right eye or left eye, grade of surgeon, and whether a complication had occurred. In the event of a complication its nature was specified as follows: incomplete capsulorrhexis, posterior capsule rupture, vitreous loss, zonule dehiscence, lost nucleus, anterior capsule tear, unplanned extracapsular cataract extraction (ECCE), corneal burn/wound leak, others (specify).

Given that patients were assigned to risk groups before their surgery, and that a complication can only be reported as having happened or not having happened, we believe that our data on complication rates and types are not subject to reporting bias or any other type of distortion.

When all the required data had been entered, the data sheet was detached and placed into a designated receptacle in the operating theatre after each cataract operation. The data were entered into a computerised database (Microsoft Access 2000) and subjected to statistical analysis. For this purpose we used the $\chi^{2}$ test (or Fisher's exact test for small data sets).

We collected data from successive phacoemulsification operations only. We did not include planned ECCE in this study. All operations included in the bag placement of an injectable hydrophilic or hydrophobic acrylic intraocular lens (IOL) or a foldable silicone IOL, except where a complication necessitated placement of a lens in the posterior sulcus or anterior chamber. A mixture of types of anaesthesia was used depending on the preference of the surgeon and the attending anaesthetist. These included topical, sub-Tenon's, and peribulbar anaesthesia as well as occasional general anaesthesia.

In order to negate the effect of a higher rate of complications by inexperienced surgeons we only included data from operations performed by consultants, fellows, and specialist registrars.

The study was conducted in two phases. In phase 1, we set out to collect data on 1000 consecutive cases in order to test the applicability of the system and analyse the results. In phase 2, we aimed to show that the results were reproducible by analysing data collected from cases over a further 3 months. This time frame yielded data on a further 441 cases.

\section{RESULTS}

We analysed data on 1000 cataract patients who had surgery between 15 November 2002 and 9 June 2003. Surgery was performed on 372 men (mean age 76.65 years) and 628 women (mean age 75.8 years), with 503 having surgery to the right eye and 497 to the left eye. The operations were performed in similar proportions by consultants $(28.4 \%)$, 


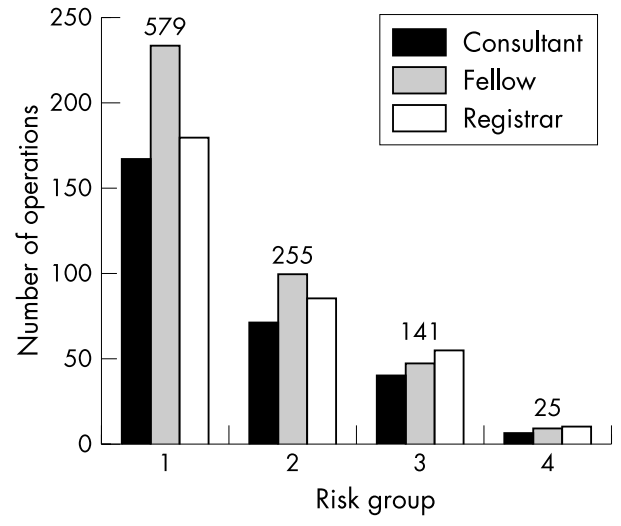

Figure 1 Number of operations by grade of surgeon and risk group.

fellows $(38.8 \%)$, and specialist registrars $(32.8 \%)$, the trend being maintained when operations were analysed as a whole and by risk group. The number of operated eyes in each risk group was 579 in group 1 (57.9\%), 255 in group $2(25.5 \%)$, 141 in group $3(14.1 \%)$, and 25 in group $4(2.5 \%)$ (fig 1). It is noteworthy that $42.1 \%$ of all operations were performed on eyes of patients carrying at least one risk factor for intraoperative complications. This represents a large number of patients who are being given information pertinent to "routine" cases when they are not "routine."

The rate of intraoperative complications was shown to be similar for all grades of surgeon (consultants $=6.69 \%$, fellows $=7.22 \%$ and specialist registrars $=7.32 \%, p=0.949$ ), thereby eliminating the issue of surgeons' experience as a complicating factor in the statistical analysis of the data.

The group specific rate of intraoperative complications increased through the risk groups $(p<0.001)$ (fig 2$)$. The rates and $95 \%$ confidence intervals $(95 \% \mathrm{CI})$ are: group 1 $4.3 \%$ (95\% CI: 2.8 to 6.3 ), group $27.4 \%$ (95\% CI: 4.5 to 11.4 ), group $313.5 \%$ (95\% CI: 8.3 to 20.2 ), group $432 \%$ (95\% CI: 14.9 to 53.5$)$.

The total complication rate was $7.10 \%$. The strong statistical significance $(\mathrm{p}<0.001)$ of the difference in complication rates between the risk groups deserves to be highlighted.

In addition to showing an increasing rate of any intraoperative complication through the risk groups, this study has established a statistically significant increase in the rates of the following types of complication through the risk groups: posterior capsule rupture (PCR), vitreous loss, incomplete capsulorrhexis, zonule dehiscence, lost nuclear

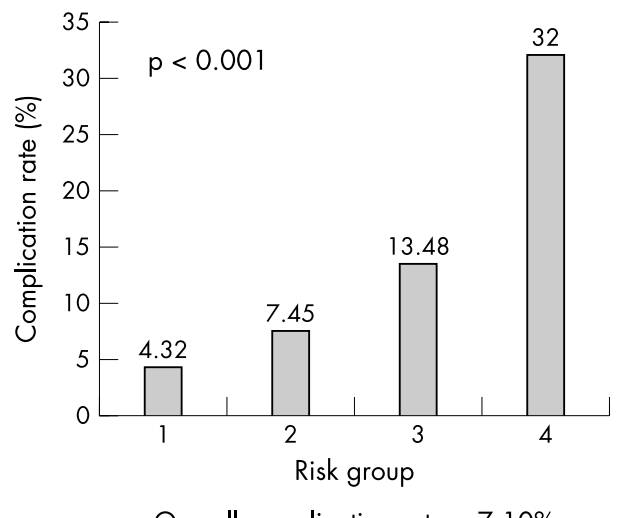

Overall complication rate $=7.10 \%$

Figure 2 Rate of complications by risk group. National Cataract Surgery Survey 1997-8 rate of any intraoperative complication: $7.5 \%$.

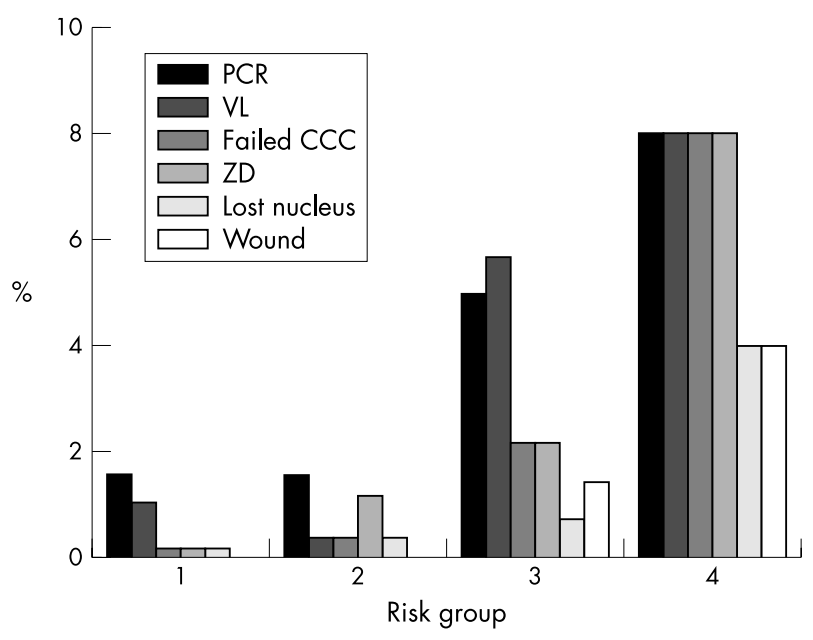

Figure 3 Rate of specific complications by risk group. PCR, posterior capsule rupture; VL, vitreous loss; CCC, continuous curvilinear capsulorhexis; ZD, zonule dehiscence; Wound, wound site thermal injury or leak.

fragments into the vitreous cavity, and corneal burn/wound leak (fig 3). These results are summarised in table 2.

The rates of the other complications were: anterior capsule tear $2.80 \%$; unplanned ECCE $0.30 \%$, iris prolapse $0.50 \%$.

None of these complications increased through the risk groups in a manner that reached statistical significance. No other types of complication were recorded.

\section{Phase 2 analysis}

Following phase 1 of the study, we collected data on consecutive patients having cataract surgery between 9 June 2003 and 12 September 2003. This yielded data on 441 cataract operations. The patients consisted of 280 women and 161 men $(63.49 \%$ and $36.51 \%$, respectively) with a mean age of 74.49 years. The distribution of right and left eyes was equal (221 right and 220 left). Specialist registrars performed most operations $(271,61.5 \%)$, with consultants $(86,19.5 \%)$ and fellows $(84,19 \%)$ sharing the remainder almost equally.

The number of operations performed in each of the risk groups was: group $1=245(55.6 \%)$, group $2=136(30.8 \%)$, group $3=51(11.6 \%)$, group $4=9(2 \%)$. These proportions are generally in line with those found in phase 1 of the study, although the actual numbers of procedures performed are much smaller in groups 3 and 4 . Therefore, only broad conclusions may be drawn from the phase 2 analysis.

The complication rates increased through the risk groups as follows: group $12.04 \%$, group $22.94 \%$, group $319.61 \%$, group $422.22 \%$.

When compared to the results from phase 1 , the rate in group 1 lies just outside the $95 \%$ CI but within the $97.5 \%$ CI; the rate in goups 2 lies outside the $95 \% \mathrm{CI}$; and the rates in groups 3 and 4 lie well within the $95 \%$ CI. The rate of posterior capsule rupture in phase 2 was group $1=1.63 \%$, group $2=1.47 \%$, group $3=3.92 \%$, group $4=0 \%$. We would point out the low number of patients in group 4 in this phase of the study and the large impact on the results that a single complication would have.

The complications that occurred in phase 2 were posterior capsule rupture and anterior capsule tear in group 1; posterior capsule rupture, anterior capsule tear, and incomplete capsulorrhexis in group 2; posterior capsule rupture, iris prolapse, corneal wound burn, zonule dehiscence, and unplanned ECCE in group 3; and lost nuclear fragments, incomplete capsulorrhexis, and unplanned ECCE in group 4. 
Table 2 Complication rates where a rise occurs through the risk groups

\begin{tabular}{|c|c|c|c|c|c|c|c|c|c|c|c|}
\hline & \multicolumn{2}{|l|}{$\begin{array}{l}\text { Group } 1 \\
\text { (0 points) }\end{array}$} & \multicolumn{2}{|c|}{$\begin{array}{l}\text { Group } 2 \\
\text { (1-2 points) }\end{array}$} & \multicolumn{2}{|c|}{$\begin{array}{l}\text { Group } 3 \\
\text { (3-5 points) }\end{array}$} & \multicolumn{2}{|c|}{$\begin{array}{l}\text { Group } 4 \\
\text { (6 points or more) }\end{array}$} & \multicolumn{2}{|l|}{ Total } & \multirow[b]{2}{*}{ p Value } \\
\hline & $(n=579)$ & (\%) & $(n=255)$ & (\%) & $(n=141)$ & $(\%)$ & $(n=25)$ & $(\%)$ & $(n=1000)$ & $(\%)$ & \\
\hline Overall & 25 & 4.32 & 19 & 7.45 & 19 & 13.48 & 8 & 32 & 71 & 7.1 & $<0.001$ \\
\hline PCR & 9 & 1.55 & 4 & 1.57 & 7 & 4.96 & 2 & 8 & 22 & 2.2 & 0.015 \\
\hline Vitreous loss & 6 & 1.04 & 1 & 0.39 & 8 & 5.67 & 2 & 8 & 17 & 1.7 & $<0.001$ \\
\hline Failed CCC & 1 & 0.17 & 1 & 0.39 & 3 & 2.13 & 2 & 8 & 7 & 0.7 & $<0.001$ \\
\hline Zonule dehiscence & 1 & 0.17 & 3 & 1.18 & 3 & 2.13 & 2 & 8 & 9 & 0.9 & $<0.001$ \\
\hline Lost nucleus & 1 & 0.17 & 1 & 0.39 & 1 & 0.71 & 1 & 4 & 4 & 0.4 & $<0.001$ \\
\hline Wound burn/leak & 0 & 0 & 0 & 0 & 2 & 1.42 & 1 & 4 & 3 & 0.3 & $<0.001$ \\
\hline
\end{tabular}

Because of the small number of operations analysed in phase 2 compared to phase 1 , the authors feel that the results should be interpreted with caution. It is our belief that the more definitive results of phase 1 have been generally supported by the phase 2 analysis (fig 4 ). A greater degree of support may be sought by applying the risk stratification system to the population groups of other hospitals. In such settings, results in agreement with those from phase 1 of this study would confirm broad applicability of our system.

\section{DISCUSSION}

The risk stratification method used in this study was based on published data and the scoring system was created de novo. The alternative approach would have been to undertake univariate regression analysis to investigate the potential risk factors for intraoperative complications in our patient population and define the odds ratio for a complication that each studied potential risk factor conferred. The odds ratio would then constitute the number of points that the particular risk factor was allocated. For example, if a diagnosis of pseudoexfoliation conferred a fivefold increased risk of intraoperative complications, then that risk factor would carry five points. A prospective study such as is the subject of this report could then be conducted. However, to investigate each of the large number of candidate risk factors in such a study would require such large patient numbers as to be possibly prohibitive, especially as some potential risk factors are quite uncommon. Furthermore, the result would be a rigid system that would be more suited to operations routinely carried out under reproducibly controlled conditions, such as cardiac surgery. However, cataract surgery is almost exclusively performed under local anaesthetic and requires a great deal of patient cooperation. Furthermore, poor positioning of the patient (for example, kyphosis in the elderly), or of the eye (for example, thyroid eye disease, chronic progressive external ophthalmoplegia) may make

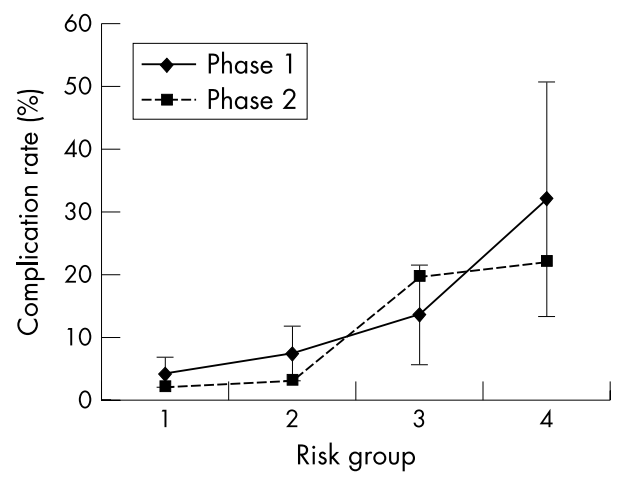

Figure 4 Complication rates in phases 1 and 2 by risk group. cataract surgery much more challenging. The diversity of circumstances under which cataract surgery is carried out necessitates that a risk stratification system should not be so rigid that it does not allow the surgeon latitude for including unforeseen or unpredictable circumstances as risk factors. In light of this, and the wealth of high quality published data on this subject, the authors chose the approach described above.

Furthermore, we did not allocate points to the risk factors based on the reported associated rates of intraoperative complications because these rates were not identical across the studies and they frequently referred only to specific complications (such as incomplete capsulorrhexis, capsular rupture, or vitreous loss) rather than all intraoperative complications, as is the case in our study.

In our scoring system, for reasons explained above, we allow for a subjective judgment of unforeseen difficulty by the surgeon. Although the potential for exploiting this element of subjectivity is clear, this study suggests that experienced surgeons would not use this as an opportunity to exaggerate the difficulty of their caseload. Indeed, this element of the system was only occasionally utilised in our study. While we anticipate a certain degree of concern regarding this component of our system, we advocate its inclusion in the expectation that data will be recorded honestly and that any skewed recording of risk would be apparent when a surgeon provides data on his/her caseload. Whether or not this part of our system is exploited in practice will only become apparent as the system is applied more widely. Under such circumstances it may be argued that the opportunity to record "miscellaneous risks" should be withdrawn. Currently, that is not the case.

In a similar way, we have kept the system deliberately simple and easily applicable in order to encourage its wider acceptability and applicability. To this end, we have relied on the common sense of our target audience rather than over defining some of the criteria used in the scoring system. This is most relevant when considering risk factors that are open to interpretation, such as corneal scarring or dense cataract. For example, we believe most surgeons would only consider that corneal scarring should score a point if it is located in the operative field and is of sufficient density to cause some degree of degradation of the view.

\section{CONCLUSION}

We have reported on a simple method for the preoperative stratification of cataract patients into groups according to their risk of intraoperative complications. The method utilises data that are easily assessed in an outpatient setting without necessitating further investigations. A risk score was found to be predictive of surgical complications.

A weighted scoring system for all risk factors in a given patient would have several benefits. Preoperative counselling 
on the chances of operative complications would be more individualised and accurate. In a teaching centre, cases suitable for trainee instruction could be identified and operating lists planned according to the experience of the surgeons present. For example, it may be possible to ensure that group 4 cases did not attend for surgery in the absence of a suitably experienced surgeon and that group l cases were scheduled for surgery on lists that are designated for teaching. This approach would increase the effective use of operating theatre time.

Of critical importance, surgeons and hospitals would be able to record their own complication rates in a way that reflects the "severity" of their caseload. This would overcome the difficulty of comparing surgical outcome data from one institution (or surgeon) with those from another. It would also allow fairer comparison with national data.

We are providing ophthalmic care in a time when informed consent is required to be based on the most accurate information available, and when surgical outcome data are increasingly required (clinical governance, risk management, appraisal) and in the public domain (www.drfoster.com). It is imperative that ophthalmologists collect such data based on patient classification. It is only in this way that such data may be fairly analysed, and patients provided with more accurate outcome data.

While acknowledging that further work may be required, we believe that this new system achieves the objectives of simplicity and broad applicability. We encourage testing and validating it more widely at other institutions. Should its applicability and function as a comparative tool be affirmed in this way, the planners and providers of health services may mandate the use of such a system as an integral part of service provision and data collection.

\section{ACKNOWLEDGEMENTS}

The authors thank Wen Xing and Catey Bunce of the statistics department of Moorfields Eye Hospital for their work on the statistical analysis of the data.

\section{Authors' affiliations}

M Muhtaseb, A Kalhoro, A lonides, Moorfields Eye Hospital at St

George's Hospital, London, UK

No financial support was received for this study.

The authors have no proprietary interest in materials used in this study.

Correspondence to: Mr Mohammed Muhtaseb, Department of Ophthalmology, 5th Floor Lanesborough Wing, St George's Hospital, Blackshaw Road, London SW17 OQT, UK; mohammedmuhtaseb@ yahoo.co.uk

Accepted for publication 7 June 2004

\section{REFERENCES}

1 www.doh.gov.uk/hes/tables/tb01001a.pdf.

2 www.statistics.gov.uk/census2001/demographic_uk.asp.

3 www.census.gov/main/www/popclock.html.

4 www.cdc.gov/nchs/data/ad/ad300ł4.pdf.

5 Desai P. The National Cataract Surgery Survey: II. Clinical outcomes. Eye 1993;7:489-94.

6 Desai P, Minassian DC, Reidy A. The National Cataract Surgery Survey 1997-8: a report of the results of the clinical outcomes. Br J Ophthalmol 1999;83:1336-40.

7 Kuchle M, Viestenz A, Martus P, et al. Anterior chamber depth and complications during cataract surgery in eyes with pseudoexfoliation syndrome. Am J Ophthalmol 2000;129:281-5.

8 Drolsum L, Haaskjold E, Sandvig K. Phacoemulsification in eyes with pseudoexfoliation. J Cataract Refract Surg 1998;24:787-92.

9 Chakrabarti A, Singh S, Krishnadas R. Phacoemulsification in eyes with white cataract. J Cataract Refract Surg 2000;26:1041-7.

10 Brazitikos P, Tsinopoulos I, Papadopoulos N, et al. Ultrasonographic classification and phacoemulsification of white senile cataracts. Ophthalmology 1999;106:2178-83.

11 Singh R, Vasavada A, Janaswamy G. Phacoemulsification of brunescent and black cataracts. J Cataract Refract Surg 2001;27:1762-9.

12 Lacalle V, Garate F, Alday N, et al. Phacoemulsification cataract surgery in vitrectomized eyes. J Cataract Refract Surg 1998;24:806-9.

13 Pinter S, Sugar A. Phacoemulsification in eyes with past pars plana vitrectomy: Case-control study. J Cataract Refract Surg 1999:25:556-61.

14 Vasavada A, Singh R. Phacoemulsification in eyes with posterior polar cataract. J Cataract Refract Surg 1999;25:238-45.

15 Osher R, Yu B C-Y, Koch D. Posterior polar cataracts: a predisposition to intraoperative capsular rupture. J Cataract Refract Surg 1990;16:157-62.

16 Berler DK. Intraoperative complications during cataract surgery in the very old. Trans Am Ophthalmol Soc 2000;98:127-32. 